\title{
APPROACH FOR THE SEMI-AUTOMATIC VERIFICATION OF 3D BUILDING MODELS
}

\author{
P. Helmholz*, D. Belton, S. Moncrieff \\ CRC for Spatial Information \\ Department of Spatial Sciences, Curtin University, GPO Box U 1987, Perth, WA, 6845, Australia, \\ (petra.helmholz, d.belton, s.moncrieff)@curtin.edu.au
}

\author{
Commission VI, WG VI/4
}

KEY WORDS: Urban, Automation, Integration, Adjustment, Updating, Change Detection, Fusion, Multisensor

\begin{abstract}
:
In the field of spatial sciences, there are a large number of disciplines and techniques for capturing data to solve a variety of different tasks and problems for different applications. Examples include: traditional survey for boundary definitions, aerial imagery for building models, and laser scanning for heritage facades. These techniques have different attributes such as the number of dimensions, accuracy and precision, and the format of the data. However, because of the number of applications and jobs, often over time these data sets captured from different sensor platforms and for different purposes will overlap in some way. In most cases, while this data is archived, it is not used in future applications to value add to the data capture campaign of current projects. It is also the case that newly acquire data are often not used to combine and improve existing models and data integrity. The purpose of this paper is to discuss a methodology and infrastructure to automatically support this concept. That is, based on a job specification, to automatically query existing and newly acquired data based on temporal and spatial relations, and to automatically combine and generate the best solution. To this end, there are three main challenges to examine; change detection, thematic accuracy and data matching.
\end{abstract}

\section{INTRODUCTION}

Over the last few decades, the number of available 3D city models and building information models (BIM) has increased. The application of such models is immense such as urban planning, disaster management, environmental simulations, infrastructure management, facility management, navigation or 3D cadastre to mention a few applications. There are initiatives to introduce standards for 3D city and BIM. One such standard is City GML by the Open Geospatial Consortium (OGC). This standard covers the regulations for visualisation, including the introduction of different Level of Details (LoD) and the positional and thematic accuracy. Following the definition of the LoD in City GML (OGC, 2012), BIMs can be interpreted as a 3D city model with the LoD4.

For the initial stage of the production of 3D city models, it is desirable to use accurate and homogenous data sources. Such data sources can be aerial laser scanning (ALS) data and pictometry (multi angle oblique imagery) often available for the whole area of interested (e.g. Au-Yeung et al, 2010). The production of 3D city models of especially the LoD3 and LoD4 is time consuming and expensive, particularly due to the high degree of manual work required. Hence, there is the natural wish of the quality assessment of these models and also to keep the $3 \mathrm{D}$ city model up-to-date. Verification is part of quality assessment and is the comparing of a topographic data set (here the city model) to the real world, as it is represented, for instance, in aerial/satellite images or laser scanning point clouds. The update includes the process of updating the existing

\footnotetext{
* Corresponding author.
}

topographic data set. A more detailed discussion of the related terminology is given in (Gerke and Heipke, 2008).

However, it is desirable for the verification/update of 3D city models to use (all) available suitable data sources. Especially, the in the last decade due to rapid developments available laser scanning technology like terrestrial laser scanners (TLS) or mobile mapping laser scanner (MLS) (Vosselman, 2009) but also imagery and traditional surveying are suitable data sources. These data sources based on different data acquisition techniques have different attributes, such as the number of dimensions, positional and thematic accuracy, precision, format of the data, and often cover only a part of the area of interest.

The layout of this paper is as follows. The background sections reviews existing approaches for the verification/update of existing 3D building models. Based on the literature review we will introduce a concept in the following section, including a detailed overview about the different data sources, and a general workflow. This is followed by an application of the concept to a simple example data set which was already implemented. The paper will close with a conclusion and outlook.

\section{BACKGROUND}

The ISO norm EN ISO 9000:2005 (1995) defines five important measures for quality of geo-data: logical consistency, completeness, positional accuracy, temporal accuracy and thematic accuracy. The logical consistency can be checked without using additional data. All other quality measures can only be derived by comparing the topographic data set to the 
real world, as it is represented for instance in laser scanning data or images. In the paper, we follow the terminology of Landes et al. (2012) and define the thematic accuracy as the accuracy of the elements, features and objects in the produced model (qualitative assessment). The positional accuracy describes the correctness of the location of the object (quantitative assessment).

The verification and updating of $2 \mathrm{D}$ topographic datasets have been explored in numerous publications, including, e.g. Helmholz et al. (2012), Göpfert et al. (2011), Ziems et al. (2011), Champion et al. (2010), Gerke and Heipke (2008), Rottensteiner (2008) and Eidenbenz et al. (2000). These approaches also use 3D information for the verification/update process. For instance, Göpfert et al. (2011) and Ziems et al. (2011) both use 3D data sets extracted from ALS or dense image matching to verify/update $2 \mathrm{D}$ road vector data sets. The verification/update processes of $2 \mathrm{D}$ topographic data sets are in general semi-automatic and offer the opportunity for the human operators to reduce the time needed for the verification/update process. The approaches are often highly specialist to one specific data sources and/or object class (Göpfert et al., 2011; Ziems et al., 2011; Champion et al., 2010; Helmholz et al., 2010; Gerke and Heipke, 2008; Rottensteiner, 2008). However, 3D topographic data sets like 3D city models face new challenges.

In this publication we will focus on land based data acquisition techniques. A good overview about more advanced point cloud processing techniques using mainly ALS data sets is given in (Vosselman, 2009).

Landes et al. (2012) proposed a qualitative (thematic accuracy) and quantitative (positional accuracy) assessment to evaluate the quality of 3D models of facades with TLS data. The quality of the facade model is a function of both, positional and thematic accuracy, whereby Landes ranks the positional accuracy higher as it is generally considered as a framework for the quality assessment of the thematic accuracy. Landes does not compare the $3 \mathrm{D}$ point cloud with the model, instead two models (reference model $B M_{i}$ and the newer model $B M_{i+l}$ ) are compared. The first step is to create the $B M_{i+1}$ in the preprocessing step which reduces noise and removes extraneous information such as trees, cars, and people. Then the TLS point cloud is segmented using RANSAC to detect planar surfaces. The planar surfaces are used afterwards for a geometric reconstruction using initially a Delaunay triangulation for the automatic extraction of façade contours. The segmentation and also reconstruction of TLS point clouds is still a research area. Hence, it is likely that these steps can introduce errors during the process of the creation of $B M_{i+1}$. These errors can lead to false alarms in the quality assessment.

Rutzinger et al. (2009) focused on the combination of ALS and MLS data to update the positional accuracy of a 2D topographic data set. Using only ALS data it is not possible to map the building outlines because the walls of the building are often covered by overhanging roofs. However, the building outlines are required for cadastral maps. Hence, vertical walls are extracted from the MLS data set. An initial region growing segmentation using Hough transformation derives segments which are classified based on planarity, inclination, wall height and width. The extracted walls are then used to update the cadastral map. This is a promising approach that offers the verification of the positional accuracy of a $2 \mathrm{D}$ topographic data set. The thematic accuracy is not considered in this publication.
The goal of Murphy et al. (2013) is the creation of BIMs of historical buildings. The core of this approach is the matching from objects to point clouds. The point clouds are extracted from TLS and imagery; the objects are given in a parametric library. Even though this work does not focus on the update of an existing data set, the core element of matching a point cloud to an object library can be also used for the verification of a $3 \mathrm{D}$ topographic data set, whereby the rather small object is replaced with 3D building models from the data set. However, the matching was developed for small objects which are part of a building; the outcome for the verification of a whole building is open.

While Murphy et al. (2013) only matches objects which are only a small part of a building, Zhang et al. (2013) matches building models based on the concepts found in the field of perception theory and Light Field descriptors. The matching results in normalised building models which are classified using the qualitative shape descriptors of Shell and Uneveness which outline integral geometrical and topographical information. However, the approach needs two building models similar to the work of Landes et al. (2012).

The methods described above are restricted to the input data. Some approaches require two building models as input data. However, the automatic creation of such building models using laser scanning or dense image matching is still on-going work. Hence, positional or thematic differences between the observed data (e.g. 3D point clouds) and the building model $B M_{i}$ can be caused by the modelling process of building model $B M_{i+1}$. Approaches using the observed $3 \mathrm{D}$ point clouds are at the moment limited to the update of 2D topographic data sets. In addition, these approaches are often limited to one specific input data source, e.g. TLS.

In this paper, we introduce an approach for the verification of a 3D building model. The approach will not be restricted to a specific data set and uses existing 3D scene operators that are controlled by prior knowledge obtained from the existing 3D building model. The approach is explained using a specific example.

\section{METHODOLOGY}

Before the workflow is presented in this chapter, the most common data sources and their characteristics for the verification of $3 \mathrm{D}$ building models will be introduced. However, the methodology will not be limited to this data set. Afterwards, the concept for the verification process will be explained.

\subsection{Data}

Data sets that are suitable for the verification of 3D building models include:

- Floor plan with building outline

- Boundary survey

- 3D point clouds acquired from ALS, TLS, MLS or imagery

Floor plans are available for a large number of buildings (e.g. emergency plans). While in general these plans are not considered in the initial creation process of the 3D city models with a LoD3, they can contribute information for the 
verification process, particularly for the thematic accuracy. Regarding LoD4 city models, an up-to-date floor plan can be used not only to verify the outside of the building but also especially the inside of the building. While the positional accuracy of floor plans can be assumed with $0.1 \mathrm{~m}$, the precision to verify/update the thematic accuracy can be assumed with less than $1 \mathrm{dm}$. An example of a floor plan is given in Figure 1.

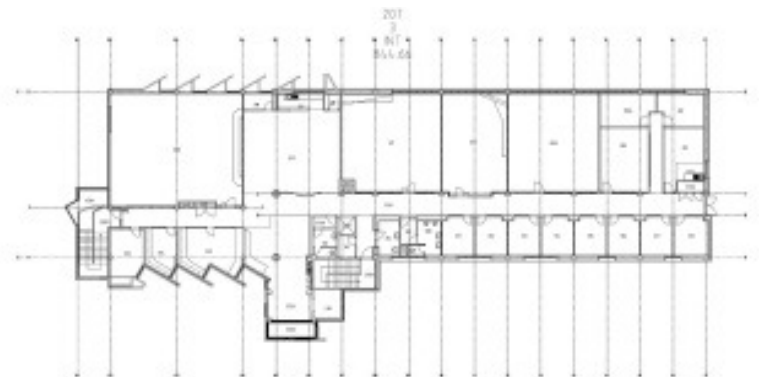

Figure 1: Sample of a floor plan $\left(3^{\text {rd }}\right.$ floor $)$.

A boundary survey is often only available in areas where recently new buildings were built. Such a boundary survey is often limited to only a small number of points which define the rough outline of the building. Therefore, this data will not be suitable to increase or correct the thematic accuracy. However, these few points are often determined with a high accuracy in the order of centimetres. This means that boundary surveys are valuable to increase the positional accuracy.

3D point clouds can be extracted from different data sources. The data sources introduce in this paper are limited to ground based or close ground platforms such as TLS systems, MLS systems or imagery taken from a small unmanned aerial vehicle (UAV).

An overview about TLS systems is given in GIM (2010). However, subsequently a number of new TLS systems have entered the market. One of these systems is the Leica $\mathrm{C} 10$ which was used to create the registered point cloud shown in Figure 2. The point cloud shows the same building as in Figure 1. In this example the colour of the points indicated the intensity of the returning light to the scanner. The Leica C10, and also a number of other scanner systems, has the ability to acquire co-located images, so the RGB information can be mapped directly to the point cloud data. In order to cover the whole building, approximately 10 different scan setups are necessary. Compared to the boundary survey a TLS data set offers a high thematic accuracy with lots of details while the positional accuracy can be assumed the same or slightly less accurate.

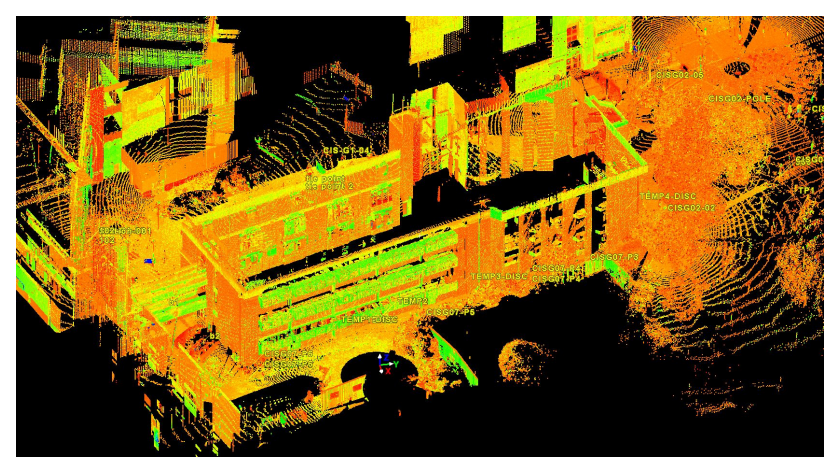

Figure 2: 3D point cloud acquired from a TLS system - here the outside of the building (colour indicates different intensity values).
TLS systems can also be used to scan the inside of a building. Figure 3 shows the scan of the third floor of the same building as visualised in Figure 1 and Figure 2 done using also a Leica C10 scanner.

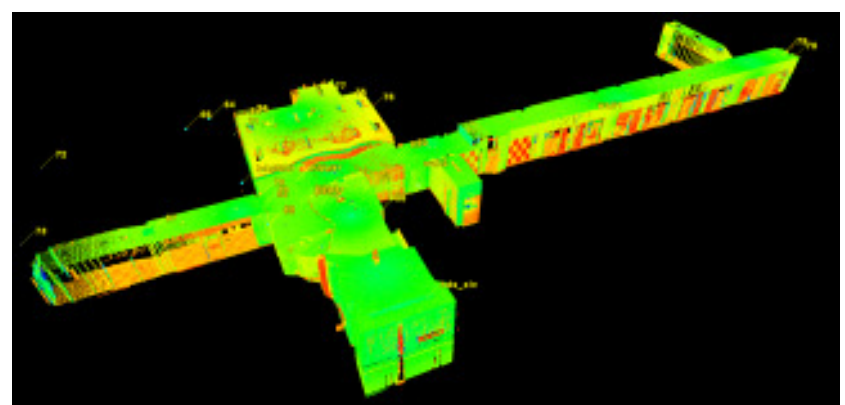

Figure 3: 3D point cloud acquired from a TLS system - here the inside of building $\left(3^{\text {rd }}\right.$ floor, colour indicates different intensity values).

3D point cloud can also be acquired from a MLS system. An overview about current MLS systems is given in (Puente et al., 2011). A MLS data set produced using the MDL Dynascan S250 is shown in Figure 4; the red arrow indicates the building of interest. While imagery was not captured in conjunction with the point cloud acquisition, it is possible with some systems to do this, allowing for RGB information to be directly applied to the point cloud data. However, compared to the TLS system in a relative short time a huge area can be scanned, but the point cloud is often less dense and the missing calibration of the MLS has a bigger effect on MLS data sets than on a TLS data set. Therefore, MLS data cannot offer the high thematic accuracy and also not a as high positional accuracy compared to the TLS dataset. The positional accuracy is highly depended on the positioning system operating together with the scanner unit, e.g. Real Time Kinematic GPS (RTK). The positional accuracy can be increased through rigorous adjustment onto a control network. Compared to boundary surveys the thematic details are higher but the positional accuracy is less.

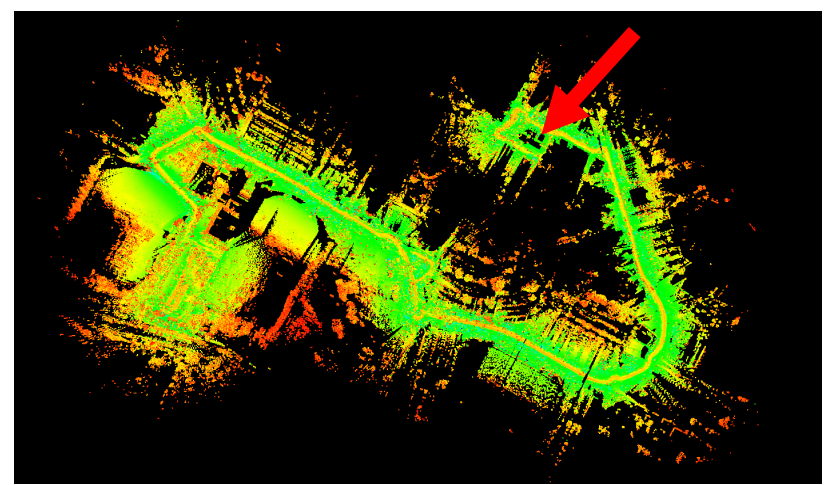

Figure 4: 3D point cloud acquired from a MLS system, the arrow highlights the building of interest (colour indicated different intensity values).

Another method to create a high dense 3D point cloud is using imagery. The use of rotor UAVs offers the possibility also to fly around the object of interest and to acquire the 3D point cloud. The range of available UAV platforms and the camera systems connected to these platforms is wide. The point cloud visualised in Figure 5 was acquired with an inexpensive Parrot AR.Drone2 UAV with the ION Air Pro 1080p HD Action Sports Video Camera. The images were processed using the approach introduced by Hollick et al. (2013). The arrow in Figure 5 highlights the flight pass around the building; the blue 
line is a missing path and the red line is separated from the other flight pass. The building is slightly deformed. It is assumed that the positional accuracy will increased if the flight loop could be closed, and the camera is calibrated prior to the flight instead of using on-the-flight-calibration. However, the point cloud is dense, and offers a photorealistic view of the building. Such small UAV systems also can be used in the inside of buildings.

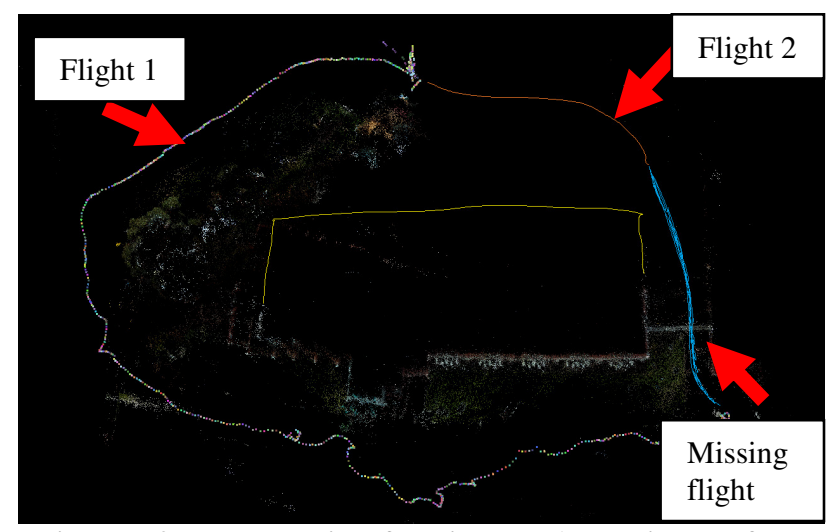

Figure 5: 3D cloud acquired from imagery (colour is taken from the imagery).

All possible input data with the expected positional accuracy and the thematic level of detail is summarised in Table 1

\begin{tabular}{|l|c|c|}
\hline Method & Positional Accuracy & Thematic Details \\
\hline Floor plan & Up to $20 \mathrm{~cm}$ & Medium (2D) \\
\hline Boundary Survey & $2-5 \mathrm{~cm}$ & Low (3D) \\
\hline TLS & $2-5 \mathrm{~cm}$ & Very high (3D) \\
\hline MLS & Up to $40 \mathrm{~cm}$ & High (3D) \\
\hline Imagery & Up to $50 \mathrm{~cm}$ & Very high (3D) \\
\hline
\end{tabular}

Table 1: Expected accuracy from the input data.

\subsection{General workflow}

For the workflow we assume that a 3D city model of buildings already exists. This can be a LoD 3 model, a LoD 4 model or simply a 2D data set like the building outline or a floor plan. An example for a building with the LoD3 is shown in Figure 6. The model was created based on a TLS data set acquired at time $t_{i}$ and is called building model $B M_{i}$. The modelling process was done semi-automatically which is time consuming.

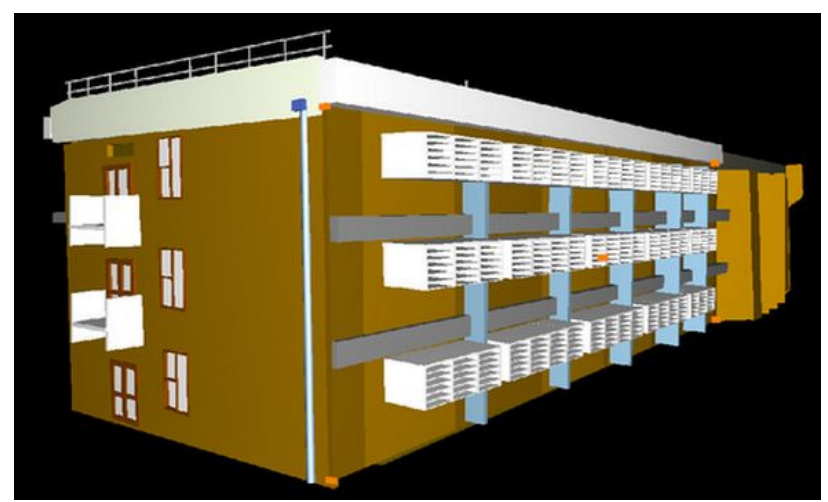

Figure 6: Building Model of previous shown 3D point cloud data sets.

The aim is to automatically look at the difference between the existing building model and the available input data (introduced in section 3.1). The workflow for the verification process is presented in Figure 7. There are two main challenges to consider; the absolute and thematic accuracy. The outcome will be then a building model $B M_{i+1}$.

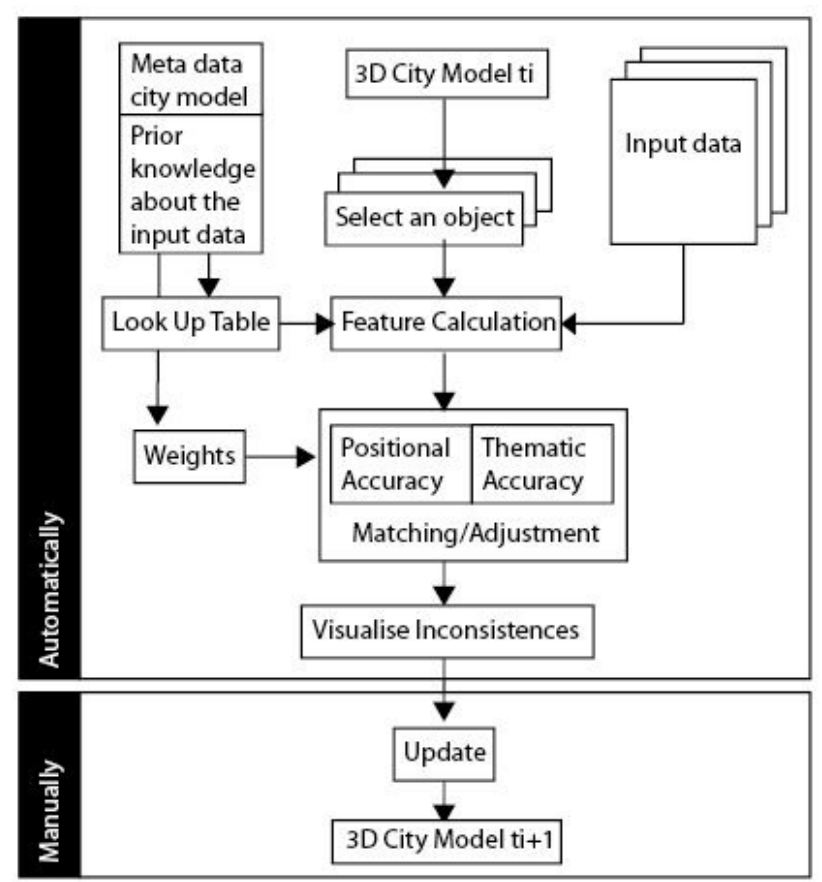

Figure 7: Workflow for the verification process.

The properties of the input data for the verification process can vary enormously with respect to the acquisition method as shown in Table 1. For instance, the dimensionality can be different (e.g. 2D vs. 3D). Another aspect is the positional and temporal accuracy, whereas in this context the positional accuracy is defined as the accuracy of the location (also shown in Table 1) and temporal accuracy as the accuracy of the model for a specific point in time, usually when the model was generated. The next aspect that should be taken into account is the precision, the scale and complexity of the existing models and acquired data sets. Furthermore, the type of available data has to be considered (pixel/point (primitive) based vs. simple features such as planes, edges vs. more complex objects like power poles automatically acquired from a point cloud). The different data properties lend themselves to different aspects of the problem. However, this information (positional accuracy, dimensionality, primitives) is in general given as prior knowledge. Prior information is also the kind of object classes contained in the topographic data set as well as further metadata like the accuracy. Hence, a look up table can be created that determines the features suitable for the matching process based on the input data set (e.g. TLS, MLS...) in combination with the information of the class of the topographic data set (e.g. building, light pole ...).

The look up table also stores the information about the weights which the different input data will have in the matching/adjustment process. For instance, a boundary survey is good for the determination of the absolute position, but lack complexity for highly detailed model generation. It is for this reason that it is important to define the desired objectives to be able to adequately select the data and to formulate adjustment constraints while processing the data. Therefore, when combining boundary survey and data acquired from TLS systems, the boundary survey would be weighted higher in the adjustment for the absolute positioning, but the TLS would 
likely be weighted higher for the thematic accuracy of the buildings i.e. façade elements. The challenge is the automatic fusing of the different data sets (more specifically the selection, weighting and interaction of all data sets) in such a way that the different data sources contribute to the optimal adjustment. Possible approaches for the matching/adjustment are DempsterShafer (Shafer, 1976), Bayesian Networks (e.g., Mitchell, 2012) or Least Square Adjustment (e.g. Niemeier, 2001).

The results of the matching/adjustment could then be visualised dynamically similar to Becker et al. (2012). For instance, outcome of the adjustment are the difference of the input and adjusted coordinates $(x, y, z)$ of the corners of the building. The user can have the opportunity to choose between different quality measures like the distance between the point coordinates or the difference in $x$ or in $y$ or in $z$ before and after the adjustment. By moving a switch up and down, the user could change the sensibility of the data which will be highlighted on the screen. For instance, the human operator wants to visualise first only difference in $x$-values before and after the adjustment higher than a $t_{\text {start }}=0.5 \mathrm{~m}$. After the model was updated, these regions could be masked out. By changing the threshold new areas could be highlighted and further updates of the object can be processed. The update is a manual iterative process till at one stage the human operator achieves a threshold $t_{\text {stop }}$ where no further update of the model is required. This threshold depends mainly on the accuracy of the updated model.

\section{EXAMPLE}

Presented in Figure 8 is a simplified example of the workflow. This example was implemented partly. Firstly, the whole workflow will be explained, and then secondly the not implemented parts will be pointed out.

The aim of this example is the automatic update of a floor plan ("the model" at time $t_{i}$ ) using a feature survey and a 3D TLS point cloud ("the input data" acquired at $t_{i+1}$ ). Suitable features for the update of the floor model using survey data and a TLS point cloud are $2 \mathrm{D}$ vertex and linear features. These features are derived automatically from the floor plan as well as the survey and the TLS data set. A matching algorithm searches through the identified features from each data set and tried to find correspondences. Since in this case the data are primarily geometrical, the correspondences are found through the relation between neighbouring and connected features (graph matching). After the correspondences were found, the data is adjusted. The elements in the different models and data are examined to look for areas of inconsistencies and errors. Based on the quality criterion "error ellipses for matching" walls that are most likely incorrect are highlighted red. The human operator only have to check the red highlighted areas instead of to inspect all model parts compared to the survey data and the TLS data set. An earlier version of this method was presented in Belton et al. (2011).

In the implementation of this example the weights and the suitable features used in the matching process were not taken from a look up table, they were fixed. Also the dynamical visualisation as mentioned in the last section is not realised yet. Instead, all objects with error ellipse axis larger than a predefined threshold are highlighted red in this example.

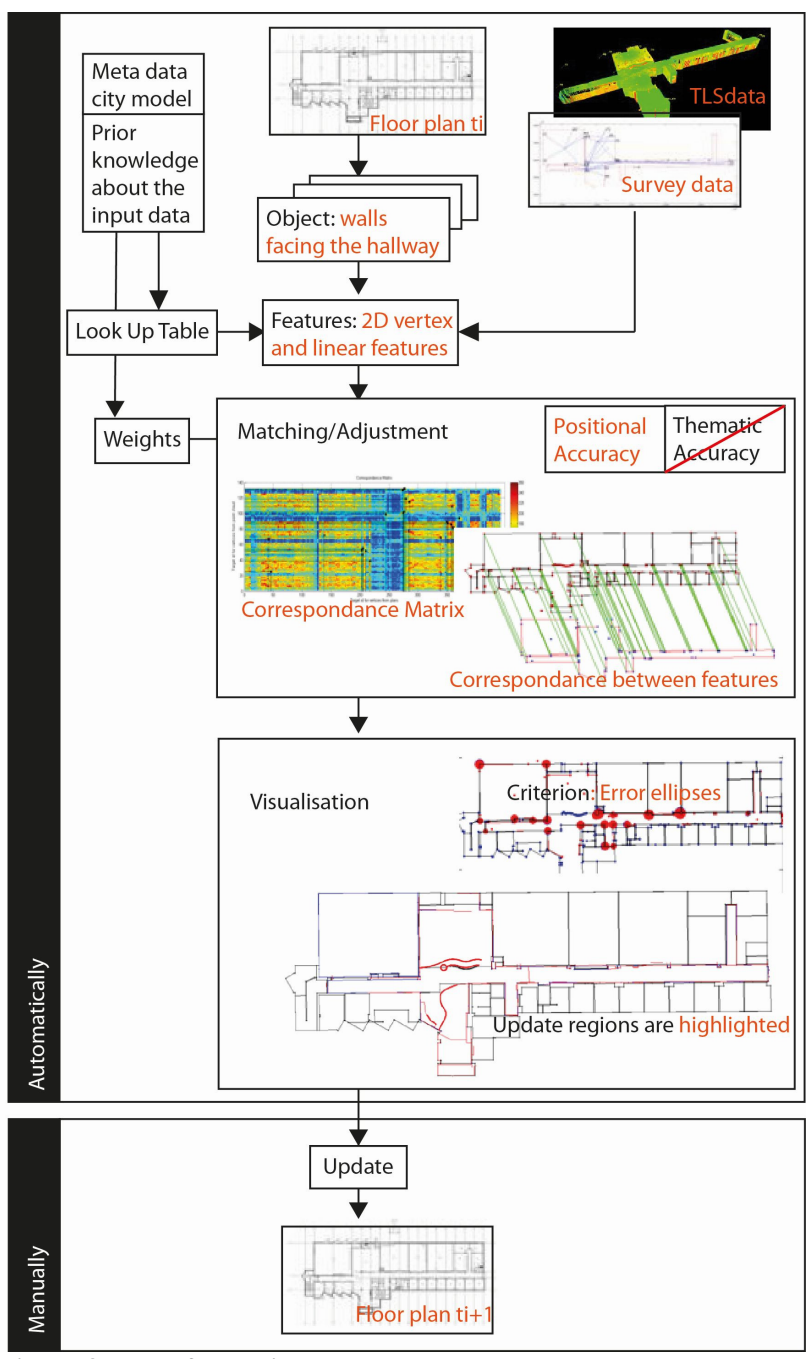

Figure 8: Workflow using a test data set.

\section{CONCLUSION AND OUTLOOK}

In this paper we introduce a concept to automatically support the verification of $3 \mathrm{D}$ building models. The concept was original designed for only 3D building models but the example showed that it also can be applied to verify $2 \mathrm{D}$ topographic data sets.

While for the presented example only simple features (2D vertex and linear features) using a high precise survey data set and a high precise/dense point cloud from a TLS system were used in a simple matching for correspondences, using less dense and less positional accurate point clouds like extracted from mobile mapping scanners is a complex problem. The features as well as the matching/adjustment process needs to be extended. At the moment, it is assumed that especially the DempsterShafer (Shafer, 1976) is suitable because it allows the easy implementation of quality measures in the decision process. The next steps includes also the implementation of the look up table as well as the dynamically visualisation of the verification results.

Also, the choice of the quality criteria for the visualisation is complex using 3D models with 3D data. It remains to be determined if simple distance measures are suitable to visualise regions of interest for the update process. 
Another challenge is to update the model semi-automatically, i.e. the systems suggests possible update scenario to the human operator who then only have to accept one suggestion and maybe includes slight changes. However, the human operator should always make the final decision regarding the update.

\section{ACKNOWLEDGMENT}

The work has been supported by the Cooperative Research Centre for Spatial Information, whose activities are funded by the Australian Commonwealth's Cooperative Research Centres Programme.

\section{REFERENCES}

Au-Yeung, B., Saad, A. \& Yigitcanlar, T., 2010. Sustainable urban infrastructure management: integration of urban computer modelling. Proceedings of the 3rd Knowledge Cities World Summit: From Theory to Practice, 16-19 August 2010, Melbourne, Victoria.

Becker, C., Pahl, M., Ostermann, J., 2012. Mono-Temporal GIS Update Assistance System based on unsupervised Coherence Analysis and Evolutionary Optimisation. ISPRS Annals, I (4): 233-238.

Belton, D., Mooney, B., Snow, T. and Bae, K-H., 2011. Automated matching of segmented point clouds to as-built planes. Proceedings of the SURVEYING \& SPATIAL SCIENCES BIENNIAL CONFERENCE 2011: 303-316.

Champion, N., D. Boldo, M. Pierrot-Deseilligny, and G. Stamon, 2010. 2D building change detection from high resolution satellite imagery: A two-step hierarchical method based on 3D invariant primitives. Pattern Recognition Letters, 31: 1138-1147.

Eidenbenz, C., C. Kaeser, and E. Baltsavias, 2000. ATOMI Automated reconstruction of topographic objects from aerial images using vectrorized map information. IntArchPhRS, 33(B3): 462-471.

Gerke, M., and C. Heipke, 2008. Image based quality assessment of road databases, International Journal of Geoinformation Science, 22(8): 871-894.

GIM, 2010. Terrestrial Laser Scanners. GIM International, August 2010.

Shafer, G., 1976. A Mathematical Theory of Evidence. Princeton University Press, 1976

Göpfert, J.; Rottensteiner, F.; Heipke, C., 2011. Using Snakes for the Registration of Topographic Road Database Objects to ALS Features. JPRS, 66(6): 858-871.

Helmholz, P., Becker, C., Breitkopf, U., Büschenfeld, T., Busch, A., Braun, C., Grünreich, D., Müller, S., Ostermann, J., Pahl, M., Rottensteiner, F., Vogt, K., Ziems, M., Heipke, C., 2012. Semi-automatic quality control of topographic datasets. PE\&RS, 78 (9): 959-972.
Helmholz, P., Rottensteiner F., Heipke, C., 2010. Automatic quality control of cropland and grassland GIS objects using IKONOS Satellite Imagery. IntArchPhRS, 38(7/B): 275-280.

Hollick, J., Belton, D., Moncrieff, S., Woods, A., Hutchison, A., Helmholz, P., 2013. IntArchPhRS, Hannover Workshop 2013 (on CD-ROM)

Landes, T., Boulaassal, H., Grussenmeyer, P., 2012. Quality Assessment of Geometric Façade Models Reconstructed from TLS data. The Photogrammetric Record, 27 (138): 137-154.

Mitchell, H.B., 2012. Data Fusion: Concepts and Ideas. Springer, 2nd ed. 2012 edition, 346 pages.

Murphy M., McGovern E., Pavia, S., Historic Building Information Modelling - Adding intelligence to laser and image based surveys of European classical architecture. ISPRS Journal of Photogrammetry and Remote Sensing, 76(2013): 89-102.

Niemeier, W., 2001. Ausgleichungsrechnung. De Gruyter Lehrbuch, 2001

Open Geospatital Consortium (OGC), 2012. OGC City Geography Markup Language (CityGML) Encoding Standard. Available at http://www.opengis.net/spec/citygml/2.0

Puente, I., González-Jorge, H., Arias, P., Armesto, J., 2011. Land-based mobile laser scanning systems: A review. IntArchPhRS 38 (5W12): 163-168.

Rottensteiner, F., 2008. Automated updating of building data bases from digital surface models and multi-spectral images, IntArchPhRS, 37(B3A): 265-270.

Rutzinger, M., Oude Elberink, S., Pu, S., Vosselman, G., 2009. Automatic Extraction of Vertical Walls from Mobile and Airborne Laser Scanning Data. IntArchPhRS 38 (3W8): 7-11.

Vosselman, G., 2009. Advanced Point Cloud Processing. Photogrammetric Week '09, pp. 137-146., Dieter Fritch (Ed.), Wichmann, Heidelberg, 2009.

Ziems, M.; Beyen, J.; Müller, S.; Roovers, S.; Heipke, C., 2011. Multiple-Model based update of Belgian reference road database. IntArchPhRS 38(4): 73-80 (on CD-ROM). 